\title{
COMMENTARY
}

\section{Diagnosis of kidney damage using novel acute kidney injury biomarkers: assessment of kidney function alone is insufficient}

\author{
Patrick T Murray* \\ See related research by Hollmen et al., http://ccforum.com/content/15/3/R121
}

\begin{abstract}
Acute kidney injury (AKI) is a syndrome that is associated with a major burden of morbidity and mortality in a variety of high risk patient populations, many of them cared for by intensivists. Following renal transplantation, delayed graft function (DGF) caused by severe acute tubular necrosis (ATN), defined by a requirement for dialysis during the initial post-transplant week, complicates postoperative management, and if prolonged ( $>14$ days), adversely affects allograft survival. Neutrophil gelatinaseassociated lipocalin (NGAL) and other novel biomarkers can detect AKI earlier than serum creatinine, and can predict AKI severity in high risk patient populations, including kidney transplant recipients. Hollmen and colleagues now demonstrate that elevated urine NGAL in deceased kidney donors is a significant risk factor for prolonged post-transplant DGF in recipients. These findings have clear implications with regard to potentially improved assessment of deceased donor suitability for potential renal allograft donation. These findings are also consistent with the growing evidence that severe ATN diagnosed by markedly elevated levels of AKI biomarkers is a useful predictor of the requirement for acute renal replacement therapy in AKI patients.
\end{abstract}

In the previous issue of Critical Care, Hollmen and colleagues [1] investigate the utility of a novel biomarker of acute kidney injury (AKI) for the pre-harvest assessment of likely outcomes of kidney transplantation from

*Correspondence: patrick.murray@ucd.ie

University College Dublin, Catherine McAuley Centre, Nelson Street, Dublin 7, Ireland deceased donors. AKI is a syndrome that is associated with a major burden of morbidity and mortality in a variety of high risk patient populations, many of them cared for by intensivists. Clinical and translational research in recent years has focused on improvements in the diagnostic and prognostic evaluation of AKI to improve care. Validation of new systems for the diagnosis and staging of AKI have been important advances, and the discovery and validation of novel biomarkers of AKI that can achieve earlier diagnosis and improve prognostic stratification have been a more recent focus in the field [2]. One such marker is neutrophil gelatinase-associated lipocalin (NGAL), which is a $25-\mathrm{kDa}$ gelatinase-bound protein that was originally characterized in neutrophils. In recent years, NGAL has been shown to be produced in the kidney following ischemic or nephrotoxic injury in both animals and humans, and has clinical utility for the early or differential diagnosis and prognostic evaluation of AKI [3-6].

Renal transplantation is a clinical setting of ischemiareperfusion injury that includes many features of laboratory experimental models of AKI, including the timed development of acute tubular necrosis (ATN), potentially permitting the use of agents for primary or secondary prophylaxis to prevent or ameliorate AKI. Delayed graft function (DGF) caused by severe ATN, defined by a requirement for dialysis during the initial post-transplant week, complicates postoperative management, and if prolonged ( $>14$ days), adversely effects allograft survival. The incidence of DGF is rising with the growing practice of accepting expanded criteria donors to increase transplantation rates. There is a limited literature investigating the utility of NGAL for the diagnostic and prognostic evaluation of AKI in kidney transplantation. Hollmen and colleagues [7], also the authors of the paper commented on here, previously found that urine NGAL from the recipient on the first post-transplant day was of moderate utility to predict DGF, particularly prolonged DGF that correlated with 
worse 1-year graft survival. Similarly, others have shown that a variety of biomarkers of renal tubular injury/ damage [8-12] or serum cystatin $C$ (a marker of kidney function/glomerular filtration rate (GFR)) [11,13] can similarly diagnose AKI secondary to DGF in the early post-transplant period better than a combination of the usual clinical parameters of kidney function (serial monitoring of serum creatinine and urine output) with known DGF risk factors (ischemia times, and so on).

Hollmen and colleagues [1] demonstrate that donor urine NGAL (but not serum NGAL) is similarly useful to help with the pre-harvest prediction of DGF after renal transplantation from deceased donors. Specifically, they studied pre-harvest serum and urine NGAL levels in 99 consecutive, deceased donors, and followed the clinical courses of their 176 kidney recipients. No other AKI biomarkers were measured. They found that high preharvest urine NGAL levels were more common in those who developed prolonged DGF. In receiver operating characteristic (ROC) curve analysis, donor urine NGAL had poor utility for prediction of DGF (area under the curve 0.595 ) or prolonged DGF (area under the curve 0.616). However, increased donor urine NGAL was a significant risk factor for prolonged DGF independent of other important predictors (expanded criteria donor status and estimated GFR). These findings have clear implications with regard to potentially improved assessment of deceased donor suitability for potential renal allograft donation. Furthermore, their findings augment the growing evidence-base suggesting the utility of biomarkers of kidney damage to provide additional diagnostic and prognostic information regarding the presence, severity, and likely course of AKI in high-risk settings, beyond what can be discerned by the use of markers of renal function (GFR and urine output) alone. Such diagnostic and prognostic accuracy has the potential to significantly improve the clinical evaluation and management of AKI, including aggressive resuscitation of patients with AKI but no evidence of kidney damage ('prerenal', reversible renal hypoperfusion resulting in decreased GFR) [4,6], and early initiation of dialysis in patients with evolving AKI caused by severe ATN (based upon biomarker evidence of severe renal tubular damage) $[3,4,14]$. Thus, the emerging use of novel AKI biomarkers of renal dysfunction and damage can offer the prospect of appropriate, early, goal-directed therapy of AKI in high risk patients, including prompt reversal where possible [6], and aggressive renal support where necessary $[3,4,14]$.

\section{Abbreviations}

AKI, acute kidney injury; ATN, acute tubular necrosis; DGF, delayed graft function; GFR, glomerular filtration rate; NGAL, neutrophil gelatinaseassociated lipocalin.

\section{Competing interests}

PTM has received research support and honoraria for scientific advisory board activity and speaking engagements from Abbott Laboratories; and research support and honoraria for scientific advisory board activity from Alere Medical. PTM is also a consultant/scientific advisory board member for FAST Diagnostics and Argutus Medical.

Published: 4 July 2011

\section{References}

1. Hollmen ME, Kyllönen LE, Inkinen KA, Lalla MLT, Merenmies J, Salmela KT: Deceased donor neutrophil gelatinase-associated lipocalin and delayed graft function after kidney transplantation: a prospective study. Crit Care 2011, 15:R121.

2. Murray P, Devarajan P, Levey A, Eckardt KU, Bonventre J, Lombardi R, HergetRosenthal S, Levin A: Criteria for AKI diagnosis and staging in different environments. Clin J Am Soc Nephrol 2008, 3:864-868.

3. Haase M, Bellomo R, Devarajan P, Schlattmann P, Haase-Fielitz A; NGAL Metaanalysis Investigator Group: Accuracy of neutrophil gelatinase-associated lipocalin (NGAL) in diagnosis and prognosis in acute kidney injury: a systematic review and meta-analysis. Am J Kidney Dis 2009, 54:1012-1024.

4. Koyner JL, Vaidya VS, Bennett MR, Ma Q, Worcester E, Akhter S, Raman S, Jeevanandam J, O'Connor MF, Devarajan P, Bonventre JV, Murray PT: Urinary biomarkers in the clinical prognosis and early detection of acute kidney injury. Clin J Am Soc Nephrol 2010, 5:2154-2165.

5. De Geus HR, Bakker J, Lesaffre EM, le Noble JL: Neutrophil gelatinaseassociated lipocalin at ICU admission predicts for acute kidney injury in adult patients. Am J Respir Crit Care Med 2011, 183:907-914.

6. Singer E, Elger A, Elitok S, Kettritz R, Nickolas TL, Barasch J, Luft FC, SchmidtOtt KM: Urinary neutrophil gelatinase-associated lipocalin distinguishes pre-renal from intrinsic renal failure and predicts outcomes. Kidney Int 2011 [Epub ahead of print].

7. Hollmen ME, Kyllönen LE, Inkinen KA, Lalla ML, Salmela KT: Urine neutrophil gelatinase-associated lipocalin is a marker of graft recovery after kidney transplantation. Kidney Int 2011, 79:89-98.

8. Parikh CR, Jani A, Mishra J, Ma Q, Kelly C, Barasch J, Edelstein CL, Devarajan P. Urine NGAL and IL-8 are predictive biomarkers for delayed graft function following kidney transplantation. Am J Transplant 2006, 6:1639-1645.

9. Hall IE, Yarlagadda SG, Coca SG, Wang Z, Doshi M, Devarajan P, Han WK, Marcus RJ, Parikh CR: IL-18 and urinary NGAL predict dialysis and graft recovery after kidney transplantation. J Am Soc Nephrol 2010, 21:189-197.

10. Hall IE, Koyner JL, Doshi MD, Marcus RJ, Parikh CR: Urine cystatin C as a biomarker of proximal tubular function immediately after kidney transplantation. Am J Nephrol 2011, 33:407-413.

11. Lebkowska U, Malyszko J, Lebkowska A, Koc-Zorawska E, LebkowskiW, Malyszko JS, Kowalewski R, Gacko M: Neutrophil gelatinase-associated lipocalin and cystatin C could predict renal outcome in patients undergoing kidney allograft transplantation: a prospective study. Transplant Proc 2009, 41:154-157.

12. Kusaka M, Kuroyanagi Y, Mori T, Nagaoka K, Sasaki H, Maruyama T, Hayakawa K, Shiroki R, Kurahashi H, Hoshinaga K: Serum neutrophil gelatinaseassociated lipocalin as a predictor of organ recovery from delayed graft function after kidney transplantation from donors after cardiac death. Cell Transplant 2008, 17:129-134

13. Hall IE, Doshi MD, Poggio ED, Parikh CR: A comparison of alternative serum biomarkers with creatinine for predicting allograft function after kidney transplantation. Transplantation 2011, 91:48-56.

14. Cruz DN, de Geus HR, Bagshaw SM: Biomarker strategies to predict need for renal replacement therapy in acute kidney injury. Semin Dial 2011, 24:124-131.

doi:10.1186/cc1025

Cite this article as: Murray PT: Diagnosis of kidney damage using novel acute kidney injury biomarkers: assessment of kidney function alone is insufficient. Critical Care 2011, 15:170. 